\title{
BMJ Open Willingness, preferred ways and potential barriers to use pre-exposure prophylaxis for HIV prevention among men who have sex with men in China
}

\author{
Simin Yu (D) , ${ }^{1}$ Wendy Cross, ${ }^{2}$ Louisa Lok Yi Lam, ${ }^{2}$ Kaili Zhang, ${ }^{3}$ Biswajit Banik, ${ }^{2}$ \\ Xianhong Li (D) , ${ }^{1}$ Honghong Wang (D) ${ }^{1}$
}

To cite: Yu S, Cross W, Lam LLY, et al. Willingness, preferred ways and potential barriers to use pre-exposure prophylaxis for HIV prevention among men who have sex with men in China. BMJ Open 2021;11:e053634. doi:10.1136/ bmjopen-2021-053634

- Prepublication history for this paper is available online. To view these files, please visit the journal online (http://dx.doi org/10.1136/bmjopen-2021053634).

Received 18 May 2021 Accepted 14 October 2021
Check for updates

(c) Author(s) (or their employer(s)) 2021. Re-use permitted under CC BY-NC. No commercial re-use. See rights and permissions. Published by BMJ.

${ }^{1}$ Xiangya School of Nursing, Central South University, Changsha, Hunan, China ${ }^{2}$ School of Health, Federation University Australia, Melbourne, Victoria, Australia

${ }^{3}$ School of Nursing, Xuzhou Medical University, Xuzhou, Jiangsu, China

Correspondence to Professor Honghong Wang; honghong_wang@hotmail.com

\section{ABSTRACT}

Objective To explore willingness and preferred ways to use HIV pre-exposure prophylaxis (PrEP), factors associated with willingness, and potential barriers to PrEP use among men who have sex with men (MSM) in Changsha, China.

Design A cross-sectional survey was conducted from 25 June to 31 August 2019. Two hundred and fifty-five MSM were recruited from three community-based organisations (CBOs) in Changsha City. Willingness and potential barriers to use PrEP were examined using researcher-created scales. Univariate and multivariate logistic regression was used to analyse the factors associated with willingness to use PrEP. P values $<0.05$ were considered significant. Setting Three MSM inclusive CBOs in Changsha, Hunan Province, China.

Participants 255 HIV-negative MSM were recruited through their CBOs with snowball sampling.

Results Less than half of the participants (43.1\%) had heard of PrEP and $15.3 \%$ were willing to use PrEP. The participants reported higher willingness to use eventdriven PrEP $(3.70 \pm 0.07)$ than daily PrEP $(2.65 \pm 0.07)$. Higher self-rated risk and fear of contracting HIV (OR: $14.47,95 \% \mathrm{Cl} 2.19$ to 95.53 ), awareness of PrEP (OR: $4.20,95 \% \mathrm{Cl} 1.64$ to 10.73 ), sharing one's own sexual orientation with parents or siblings (OR: $2.52,95 \% \mathrm{Cl}$ 1.54 to 7.20 ) and having a university education or above (OR:0.29, 95\% Cl 0.12 to 0.72 ) were associated with willingness to use PrEP. Only $12.2 \%$ of the sample was concerned about potential barriers to PrEP use. Conclusion Efforts to improve awareness and knowledge of PrEP, teach self-evaluation of HIV infection risk and provide social and emotional support for MSM are needed to scale up PrEP implementation in China.

\section{INTRODUCTION}

HIV/AIDS is a major infectious disease that seriously endangers people's health. Data from the Joint United Nations Program on HIV/AIDS showed that 38.0 million people were living with HIV and 1.7 million people were newly infected with HIV globally in 2019. ${ }^{1}$ In China, at the end of October 2019, over 958000 people were living with HIV and
Strengths and limitations of this study

- The pre-exposure prophylaxis (PrEP) willingness scale and potential barriers to use PrEP scale were adapted after the qualitative outcome analysis component of this mixed methods study, making the scale more appropriate for the Chinese men who have sex with men (MSM) population.

- This study investigated the preferred ways of taking and potential barriers to using PrEP, providing guidance for PrEP implementation among MSM in China.

- This study was conducted at three MSM-friendly community-based organisations in nine districts in Changsha City, ensuing accessibility for MSM, a hard-to-reach population.

- The relatively small sample size of this study may potentially limit the generalisability of the results to other regions.

over 131000 new cases were reported from January to October 2019. Among the new cases, heterosexual transmission accounted for $73.7 \%$, while male homosexual transmission accounted for $23.0 \% .^{2}$ The prevalence of HIV infection among Chinese men who have sex with men (MSM) has steadily increased from $5.5 \%$ in 2009 to $7.8 \%$ in 2017. ${ }^{3}$ Although many strategies (eg, safe sex education, issuing condoms, free HIV testing and medical male circumcision) have had a positive impact on reducing new HIV infection, the epidemic of HIV infection among MSM has not been adequately controlled. Multiple interventions must be integrated to control this epidemic, including the addition of biomedical interventions to traditional behavioural interventions. ${ }^{4}$ Additional safe and effective approaches to HIV prevention are urgently needed.

Pre-exposure prophylaxis (PrEP) is a widely recognised and promising strategy for HIV prevention. PrEP is the use of oral tenofovir 
disoproxil fumarate (TDF), coformulated TDF/emtricitabine (TDF/FTC) or coformulated TDF/lamivudine (TDF/3TC) by HIV-negative people to prevent HIV acquisition. ${ }^{5}$ This prevention method has been recommended by the WHO, which has emphasised the daily use of antiretrovirals in HIV-uninfected people to prevent HIV infection (including MSM) since 2012. ${ }^{5}$ In 2015, the WHO launched evidence-based guidelines recommending that anyone at substantial risk of contracting HIV should be offered PrEP. ${ }^{6}$ Moreover, in 2019, WHO broadened its recommendations on 2+1+1 event-driven oral PrEP use for MSM (two pills, which serve as the loading dose, between 2 and 24 hours in advance of sex; a third pill 24 hours after the first two pills are taken and a fourth pill 48 hours after the first two pills are taken). ${ }^{7}$ Over 50 countries have incorporated PrEP into their health and service systems ${ }^{8}$; of these countries, 10 have implemented nationwide programmes and others are conducting limited demonstration small sample projects, exploring the acceptability and costs of PrEP, for example. ${ }^{9}$

In 2019, the Chinese Center for Disease Control and Prevention (CDC) issued a document titled 'Implementation of the program to stop the spread of AIDS (20192022), which recommended pilot work on PrEP for populations such as MSM, the formulation of improved policies to promote and manage PrEP use and the gradual expansion of such policies. ${ }^{10}$ On 11 August 2020, the Chinese National Medical Products Administration approved TDF/FTC tablets for PrEP. ${ }^{11}$ To prepare for a rollout of PrEP, it is crucial to understand willingness to use PrEP and associated factors among Chinese MSM.

Previous studies in China (including mainland China, Hong Kong and Taiwan) have revealed wide variation in willingness to use oral PrEP (ranging from $7.7 \%$ to $84.9 \%$ ). ${ }^{12-23}$ Variations in willingness to use PrEP were affected by the way it was measured, with some researchers using single items and others using multiple items/scales. ${ }^{12-23}$ The availability and knowledge of PrEP have changed over time, and different circumstances in different countries could impact the measurement of willingness to use PrEP. A new comprehensive tool with content pertaining to the Chinese context is needed to measure the willingness of Chinese MSM to use PrEP.

This study aimed to investigate willingness and preferred ways of using PrEP with a researcher-designed 19-item scale as well as other associated factors from several contexts. This study also investigated concerns about potential barriers to PrEP use with a researcherdesigned nine-item scale. The findings of this study could provide evidence for recommendation-making and national decision-making with respect to PrEP implementation among MSM in China.

\section{METHODS}

\section{Study design and participants}

A cross-sectional study was carried out between 25 June and 31 August 2019, in Changsha City, the capital city of Hunan Province. Potential participants were recruited through three gay-friendly community-based organisations (CBOs) for MSM services. These three organisations were health consulting service centres, and the participants were service users. These CBOs were located in three districts in well-concealed positions and supervised by the CDC of Hunan Province. These organisations were respectively founded in 2008, 2011 and 2013 and have extensive experience in consulting, peer education and testing for HIV. In previous years, they have undertaken approximately $80 \%$ of all HIV testing of MSM in Hunan Province.

Participants were eligible if they were at least 16 years old; assigned male sex at birth; self-defined their sexual orientation as gay, bisexual or other MSM; sexually active with other men in the past year; and self-reported being HIV-negative. The sample size was calculated using the formula $\mathrm{n}=\frac{\frac{\mu_{\alpha}^{2}}{2} \pi(1-\pi)}{\delta^{2}}$, where $\alpha=0.05$, and $\pi$ was willingness to accept PrEP (considered to be 0.6). ${ }^{12} 131724$ In this calculation, $\delta$ was determined to be $6 \%$ (usually $0.1 \pi$ ); therefore, the intended sample size was $256(1.96 \times 19.6 \times 0$ $.6 \times 0.4 / 0.06 / 0.06=256)$.

\section{Measurements}

\section{General information}

A comprehensive battery of demographic questions was asked, including questions regarding age, ethnicity (Han or other), educational background (high school or below, or university/college or above), occupational status (fulltime student or employed), monthly average income and household registration type (rural or urban). Current relationship status (single, married or living with a female, or in an intimate relationship with a male), sexual orientation (homosexual, bisexual or not sure) and sexual preference (men only or both men and women) were reported as well. Participants were also asked about when their last HIV tests occurred (never tested, $<6$ months, 6-12 months or $>12$ months ago), sexually transmitted infection (STI) diagnoses in the past year, self-rated risk and fear of contracting HIV $(0,<25.0 \%, 25.0 \%-49.0 \%$, $50.0 \%-75.0 \%$, or $>75.0 \%$ ) and perception of HIV infection risk among friends who are MSM $(0,<25.0 \%, 25.0 \%-$ $49.0 \%, 50.0 \%-75.0 \%$, or $>75.0 \%)$. Another measured risk factor was illicit substance use in the past 6 months (ie, rush/poppers, ketamine, crystal, heroin, cocaine/ crack and ecstasy/methylenedioxy-methylamphetamine (MDMA)/molly).

\section{Sexual behaviours}

Questions regarding sexual behaviours in the past 6 months included the participant's number of regular male partners (count), number of casual male partners (count), female sexual partners (yes or no), instances of receptive and/or insertive condomless anal sex (CAS; yes or no), number of casual partners when having CAS (count), HIV-positive male partners (yes, no or not sure) and exchange of sex for money (yes or no). 
Table 1 Willingness to use PrEP among MSM

Items

Mean \pm SD

1. Considering my current situation, I am willing to take PrEP pills to avoid HIV infection.

$3.60 \pm 0.07$

2. If PrEP reduced my risk of HIV infection, I would prefer to take it before and after sex.

$3.70 \pm 0.07$

3. If PrEP reduced my risk of HIV infection, I would prefer to take one pill per day.

$2.65 \pm 0.07$

4. I am willing to use PrEP pills as soon as they are available.

$3.18 \pm 0.07$

5. I find it inconvenient to go to a designated hospital to get a prescription for PrEP pills. (reversed score)

$2.72 \pm 0.06$

6. I do not think that I need to take PrEP pills. (reversed score)

$3.31 \pm 0.07$

7. I am willing to take PrEP pills because they reduce my anxiety or fear of unsafe sex.

$3.61 \pm 0.06$

8. Even if I insist on using condoms, I am still willing to take PrEP pills because they reduce my fear of AIDS/HIV.

$3.34 \pm 0.06$

9. I am willing to pay for PrEP pills.

$3.20 \pm 0.06$

10. I only use PrEP pills when they are free or covered by Medicare.

$3.00 \pm 0.07$

11. I am willing to take PrEP pills even if they are not $100 \%$ effective in preventing HIV.

$3.55 \pm 0.06$

12. I am willing to take PrEP pills even if there are mild side effects.

13. I am willing to take PrEP pills even if I need regular tests for HIV and STDs after taking them.

$3.53 \pm 0.06$

14. I am willing to take PrEP pills even if they require regular hospital check-ups.

$3.41 \pm 0.06$

15. I would be more willing to take PrEP pills if I knew how they work.

16. I will wait until someone else has taken PrEP pills before I take PrEP. (reversed score)

17. If PrEP pills were tested and found to be effective in Chinese gay people, I would be more willing to use them.

18. I would be more willing to take PrEP pills if my partner/friend told me he was taking them.

$4.09 \pm 0.05$

19. If my doctor recommends PrEP pills, I will take them.

MSM, men who have sex with men; PrEP, pre-exposure prophylaxis; STD, sexually transmitted diseases.

\section{Awareness of PrEP}

Participants were asked whether they had heard about PrEP and, if so, where they had heard about it (eg, online/ internet, non-governmental organisation (NGO)/CBO, sex partner, doctor, friend, family member, television/ radio or other). They were also asked whether they had ever taken PrEP and, if so, how they took it $(2+1+1 /$ eventbased dosing, one pill everyday or irregularly). Participants were also asked about additional sources of PrEP awareness, such as knowing someone who had taken PrEP. Those who had heard about PrEP were also asked whether they were initially consulted about PrEP by a doctor or staff from CBOs or ever professionally informed of PrEP; if they had been, they were asked about their basic knowledge of PrEP (eight related questions). Finally, all participants were asked how effective they thought PrEP was in preventing HIV infection (very effective, having some preventive effect, ineffective or not sure).

\section{Willingness and preferred ways of using PrEP}

Participants were asked to rate how likely they were to take PrEP on a 19-item scale with each five-point Likert item ranging scored from 'extremely unlikely' to 'extremely likely'. This comprised the quantitative step of the present mixed methods study. The 19-item scale was based on a seven-item scale developed by Holt $e t a l^{25}$, taking PrEP availability in Changsha, China into consideration and combined with the thematic analysis from the qualitative portion of the present study (table 1).
Of the 19 items, two concerned preferred ways of using PrEP: 'If PrEP reduced my risk of HIV infection, I would prefer to take it before and after sex' and 'If PrEP reduced my risk of HIV infection, I would prefer to take one pill per day'. Combining the scores of three experts, the scale's item-level content validity index (I-CVI) was 1.00 for 17 items and 0.67 for two items. The scale-level content validity index (S-CVI) scores were 0.985 for $\mathrm{S}-\mathrm{CVI} /$ universal agreement (S-CVI/UA) and 0.965 for S-CVI/average (SCVI/Ave). ${ }^{26}$ After analysis with SPSS, the Cronbach's $\alpha$ was found to be 0.905 . Each item was scored from 1 to 5 . The mean of the scores on all the items formed the scale score. Participants who scored equal to or higher than four points were considered willing to use PrEP.

\section{Potential barriers to PrEP use}

Participants were asked to rate their concern about potential barriers to PrEP use on a nine-item scale scored from 1 'extremely unlikely' to 5 'extremely likely'. Combining the scores of three experts, the I-CVI of this scale was 1.00 for all items, the S-CVI/UA was 1.00 and the SCVI/ Ave was $1.00{ }^{26}$ After analysis with SPSS, the Cronbach's $\alpha$ was found to be 0.894 . The mean of the scores on all the items formed the scale score. Participants who scored equal to or higher than four points were considered to be concerned about the potential barriers to PrEP use (table 2). 
Table 2 Concerns about potential barriers to using PrEP

\begin{tabular}{|c|c|}
\hline Items & Mean \pm SD \\
\hline 1. I worry that people will think I am HIV positive or being treated for AIDS. & $3.07 \pm 0.07$ \\
\hline 2. I worry that people will find out I am gay. & $2.97 \pm 0.07$ \\
\hline 4. I worry that people will think I am a sex worker. & $2.78 \pm 0.08$ \\
\hline 5. I do not want anyone to know I am on PrEP. & $3.54 \pm 0.07$ \\
\hline 7. Using PrEP pills makes me feel embarrassed. & $2.65 \pm 0.07$ \\
\hline 8. Asking my doctor for a prescription for PrEP pills would make me feel embarrassed. & $2.77 \pm 0.07$ \\
\hline $\begin{array}{l}\text { 9. Compared with hospitals/designated pharmacies, I prefer to obtain PrEP drugs from ordinary pharmacies/ } \\
\text { gay working groups or purchase them myself abroad. }\end{array}$ & $3.37 \pm 0.07$ \\
\hline
\end{tabular}

PrEP, pre-exposure prophylaxis.

\section{Data collection}

Recruitment for the study took place at three CBOs. If MSM came to the CBO, the staff would mention to them that there was a PrEP research project underway, and whether they were willing to meet the researchers for further information. If MSM were willing to meet the researchers, the CBO staff would lead them to an office, where the researchers were. The CBO staff would leave the room, allowing for private conversation between the participant and the researcher. Potential participants were informed about the background, aim and method of the study and could decide to participate in the research or not.

The online survey was designed with 'Wenjuanxing' software that helps with online survey collection. Anonymous survey data were collected through several steps. A quick response code (QR code, a type of matrix barcode/ two-dimensional barcode) generated by Wenjuanxing was provided by the researchers; when potential participants scanned the QR code on their smartphone, they were taken to a homepage that detailed the background and aims of the present study. Each potential participant completed an online informed consent form if they agreed to participate. Completion of a demographic screener in the online survey helped to determine eligibility. The participants were provided with a brief introduction to PrEP in the online survey, including the principles of prevention, evidence concerning the effectiveness of PrEP, two recommended ways of using PrEP (daily use and event-driven use) and the side effects of using PrEP. PrEP is available in Changsha City with a doctor's prescription from a HIV/AIDS-specific hospital and costs 1980 RMB (approximately US\$291) for one bottle with 30 tablets. The participants who provided informed consent clicked through to an online survey that took approximately $30 \mathrm{~min}$ to complete. A process of checking conflicting answers was embedded into the online survey system to control the quality of the participants' responses. Participants filled in the surveys on their own. On completion of the survey, the participants were given a 50 RMB (approximately US\$7.5) allowance for their time and transportation. The researchers were in the same room as the participants throughout the questionnaire completion process, offering help if the participants felt confused about scanning the $\mathrm{QR}$ code or the way the questions were phrased.

\section{Statistical analysis}

Descriptive analyses were carried out for all variables. Bivariate analyses were used to assess bivariate relationships between independent variables. Factors that were significantly associated with willingness to use PrEP in the univariate regression analysis $(\mathrm{p}<0.05)$ were purposely selected and further explored using multivariate logistic regression analysis to determine which of these variables were independently associated with willingness to use PrEP. Statistical significance was set at $\mathrm{p}<0.05$. All statistical analyses were conducted using SPSS V.25.0. Incomplete questionnaires were deleted and excluded from the analysis.

\section{Patients and public involvement}

No patients were involved in the design of this study. Three CBOs helped with recruitment.

\section{RESULTS}

\section{Sociodemographic characteristics of participants}

A total of 287 MSM were informed about the study and 256 MSM agreed to participate. The response rate was $89.2 \%$. The survey was conducted with 256 men who met the inclusion criteria and consented to participate. After the removal of one uncompleted questionnaire, the data of 255 participants were included in the final analysis. The ages of these participants ranged from 18 to 45 years (mean $=24.51$ years, $\mathrm{SD}=0.38$ years). The majority of the participants were of Han ethnicity $(94.5 \%)$ and from a rural household $(56.9 \%)$. Out of the 255 participants, 169 men $(66.3 \%)$ were employed and $33.7 \%$ were fulltime students. Most of the participants were educated 
at the college level $(70.2 \%)$, and $48.2 \%$ indicated a monthly income $<4000$ RMB (approximately US\$587). Over half of the participants were single $(54.9 \%)$, identified themselves as homosexual $(72.9 \%)$ and had sex with men exclusively $(72.9 \%)$. In addition, $40.4 \%$ of the participants were in an intimate relationship with a man $(40.4 \%)$ and $4.7 \%$ were married or living with a woman. Smaller percentages identified as bisexual $(25.1 \%)$ or were not sure of their sexuality (2.0\%) (table 3).

Over three-fifths of the sample $(65.1 \%)$ had been tested for HIV at least once, and over half of the sample (55.3\%) reported having had an HIV test within the last 6 months. In terms of STI screening, 141 participants $(55.3 \%)$ had never been tested and eight participants $(3.1 \%)$ had been diagnosed with an STI. The participants' self-rated risk and fear of contracting HIV were low: $20.8 \%$ believed they were at no risk, and $58.0 \%$ believed their risk was below $25.0 \%$. Only $11.7 \%$ of the sample thought their gay friends had a risk of HIV infection greater than $75.0 \%$. The most commonly used substance among participants was Rush (poppers; $23.1 \%$ ), and $72.5 \%$ of the participants declared no substance use.

\section{Sexual behaviours}

In terms of sexual behaviours, 132 participants reported only one regular partner and 74 participants reported two or more casual partners. Four participants reported having had commercial sex and 30 participants had female sex partners. Furthermore, $36.5 \%$ of the sample reported having had CAS, and $41.9 \% \quad(n=93)$ of these participants reported having had CAS with casual partners. Eight participants reported HIV-positive partners, and 105 participants were unaware of their partners' serostatuses.

\section{Awareness of PrEP}

Nearly half of the participants $(n=145,43.1 \%)$ reported that they had never heard of PrEP prior to this study. Only one participant reported having ever taken PrEP pills, and he took them irregularly. Those who had heard about PrEP cited the internet (66.2\%), CBOs (37.2\%) and friends (17.9\%) as their main sources of information about PrEP. Seventy-one participants reported that they had been initially consulted about or informed of PrEP by the staff of a CBO $(42.1 \%)$ or by a doctor or nurse $(6.9 \%)$.

In general, the participants had insufficient knowledge of PrEP. The two questions with the highest correct response rates concerned the effectiveness of PrEP, and the key to PrEP's effectiveness is adherence. Of the participants, $41.2 \%$ viewed PrEP as a very effective strategy and $53.3 \%$ thought that PrEP may have some effect on HIV prevention. Three participants thought that PrEP was totally useless (table 4 ).

\section{Willingness and preferred ways to use PrEP}

The mean scores on the individual scale items regarding willingness and preferred ways to use PrEP ranged from
Table 3 Demographic characteristics of the study sample

\begin{tabular}{clc}
\hline Character & Frequency & Percentage \\
\hline Age & & \\
$18-22$ & 127 & 49.8 \\
$23-27$ & 61 & 23.9 \\
$\geq 28$ & 67 & 26.3 \\
Ethnicity & & \\
Han & 241 & 94.5 \\
Others & 14 & 5.5 \\
\hline
\end{tabular}

Educational background

$\begin{array}{llr}\text { Junior high school or below } & 14 & 5.5 \\ \text { Senior high school } & 49 & 19.2 \\ \text { University/college } & 179 & 70.2 \\ \text { Master degree or above } & 13 & 5.1 \\ \text { Occupational status } & & \\ \text { Full-time student } & 86 & 33.7 \\ \text { Employed } & 169 & 66.3\end{array}$

Average monthly income

\begin{tabular}{llr} 
1-1999 RMB & 59 & 23.1 \\
2000-3999 RMB & 64 & 25.1 \\
4000-5999 RMB & 83 & 32.5 \\
6000-7999 RMB & 19 & 7.5 \\
8000 RMB or above & 30 & 11.8 \\
\hline
\end{tabular}

Current living conditions

Very stable (living in a place $75 \quad 29.4$

purchased by oneself or by

parents)

Relatively stable (renting a $\quad 178 \quad 69.8$

room or dormitory)

Unstable (no permanent $\quad 2$
residence)

Household registration type

$\begin{array}{lll}\text { Rural } & 145 & 56.9 \\ \text { Urban } & 110 & 43.1\end{array}$

\begin{tabular}{llr} 
Current relationship status & & \\
Single & 140 & 54.9 \\
$\begin{array}{l}\text { Married or living with a } \\
\text { female }\end{array}$ & 12 & 4.7 \\
\hline
\end{tabular}

In an intimate relationship $\quad 103 \quad 40.4$

with a male

\begin{tabular}{llr} 
Sexual orientation & & \\
Homosexual & 186 & 72.9 \\
Bisexual & 64 & 25.1 \\
\hline Not sure & 5 & 2.0
\end{tabular}

With whom have you talked about your sexual orientation? (multiple choice)

\begin{tabular}{llr} 
No one & 38 & 14.9 \\
Parents or siblings & 47 & 18.4 \\
Wife or girlfriend & 8 & 3.1 \\
\hline
\end{tabular}

Continued 


\begin{tabular}{lll} 
Table 3 Continued & & \\
\hline Character & Frequency & Percentage \\
\hline Friends & 193 & 75.7 \\
$\quad$ Colleagues or classmates & 69 & 27.1 \\
$\begin{array}{l}\text { Sexual preference } \\
\text { Men only }\end{array}$ & 186 & 72.9 \\
Both men and women & 69 & 27.7 \\
\hline
\end{tabular}

1.37 to 4.68 . The mean score on the overall 19-item scale was $3.41 \pm 0.61$; this indicated that, on average, the MSM in this study fell slightly above neutral in their endorsement of willingness to use PrEP, as each item was scored from 1 ('extremely unlikely') to 5 ('extremely likely'). A score of four points or higher indicated willingness to use PrEP. Overall, 39 participants $(15.3 \%)$ had mean scores of four points or higher (were willing to use PrEP). The majority of the participants $(84.7 \%)$ were unwilling to use or neutral about PrEP (table 1).

The participants' scores on each item indicated that Chinese MSM would be more willing to use PrEP if it was tested in Chinese MSM and recommended by their doctors. They were also more willing to take PrEP if they knew how it worked or knew that their friends were taking PrEP. The participants' mean scores on two items concerning preferred ways to use PrEP indicated that daily PrEP use $(2.65 \pm 0.07)$ was less preferable than eventdriven use $(3.70 \pm 0.07)$.

\section{Concerns regarding potential barriers to PrEP use}

The mean scores on the individual scale items regarding potential barriers to PrEP use ranged from 1.00 to 5.00 with a mean score of $3.03 \pm 0.51$; this indicated that, on average, the MSM in this study fell slightly above neutral in their endorsement of concerns regarding the potential barriers to PrEP use. Overall, 31 participants had a mean score of $\geq 4$. Only $12.2 \%$ of the participants were concerned about potential barriers to PrEP use, while the remaining $87.8 \%$ were not (table 2 ).

Of the nine items on this scale, the mean scores on four items were above three points. The lowest mean score was $2.77 \pm 0.07$ for 'asking my doctor for a prescription for PrEP pills would make me feel embarrassed'. The highest mean score was $3.54 \pm 0.07$ for 'I do not want anyone to know I am on PrEP'.

\section{Factors associated with willingness to use PrEP}

According to the univariate logistic regression, participants who had university/college or higher education were less willing to use PrEP (OR: $0.46,95 \%$ CI 0.22 to 0.94). Participants were more willing to use PrEP if they had told their parents or siblings about their sexual orientation (OR: 2.68, 95\% CI 1.25 to 5.72), reported a high level of risk and fear of contracting HIV $(>75.0 \%$; OR: 16.00, 95\% CI 2.92 to 87.77), had ever heard of PrEP (OR: 2.30, 95\% CI 1.33 to 6.46). In the univariable model, participants who were not sure about the effectiveness of
Table 4 Awareness and knowledge of PrEP

\begin{tabular}{lcc}
\hline Character & Frequency & Percentage \\
\hline Awareness of PrEP $(n=255)$ & \multicolumn{3}{l}{} \\
No & 110 & 43.1 \\
Yes & 145 & 56.9 \\
Information resources (multiple choice, $n=145)$ \\
The internet & 96 & 66.2 \\
CBOs & 54 & 37.2 \\
Sexual partners & 6 & 4.1 \\
VCT clinic doctors & 6 & 4.1 \\
Friends & 26 & 17.9 \\
TV & 4 & 2.8
\end{tabular}

Initially consulted about or informed of $\operatorname{PrEP}(n=145)$

$\begin{array}{llr}\text { No } & 74 & 51.0 \\ \text { Yes, by the staff of a CBO } & 61 & 42.1 \\ \text { Yes, by a doctor or a nurse } & 10 & 6.9 \\ \text { (Knowledge of PrEP } n=71 \text { ) } & & \end{array}$

To what extent does PrEP prevent HIV infection if it is used strictly according to medical advice?

\begin{tabular}{|c|c|c|}
\hline Approximately 30\% & 3 & 4.2 \\
\hline Approximately $50 \%$ & 3 & 4.2 \\
\hline Approximately $80 \%$ & 9 & 12.7 \\
\hline Over $90 \%$ & 50 & 70.4 \\
\hline I do not know & 6 & 8.5 \\
\hline \multicolumn{3}{|c|}{ How many people worldwide are currently using PrEP? } \\
\hline Less than or equal to 50000 & 7 & 9.8 \\
\hline $50001-100000$ & 2 & 2.8 \\
\hline $100001-150000$ & 1 & 1.4 \\
\hline $150001-200000$ & 1 & 1.4 \\
\hline More than 200000 & 18 & 25.4 \\
\hline I do not know & 42 & 59.2 \\
\hline
\end{tabular}

What is the approximate percentage of every 100 PrEP users that experience common drug side effects, such as headaches, nausea, and weight loss?

\begin{tabular}{llr}
$10 \%$ & 23 & 32.4 \\
$20 \%$ & 4 & 5.6 \\
$30 \%$ & 1 & 1.4 \\
$40 \%$ & 2 & 2.8 \\
$50 \%$ & 3 & 4.2 \\
Over 50\% & 3 & 4.2 \\
I do not know & 35 & 49.3 \\
\hline
\end{tabular}

How often do PrEP users typically need to be followed up to monitor medication side effects, blood test results, and STDs?

\begin{tabular}{llr} 
Every month & 10 & 14.1 \\
Every 3 months & 28 & 39.4 \\
Every 6 months & 3 & 4.2 \\
I do not know & 30 & 42.3 \\
\hline
\end{tabular}

Continued 
Table 4 Continued

Character

Frequency Percentage

For PrEP users, which of the following body parts need to be regularly tested for gonorrhoea and chlamydia?

\begin{tabular}{|c|c|c|}
\hline Rectum only & 3 & 4.2 \\
\hline Urethra only & 4 & 5.6 \\
\hline Pharynx only & 1 & 1.4 \\
\hline All of the above & 25 & 35.2 \\
\hline I do not know & 38 & 53.6 \\
\hline \multicolumn{3}{|c|}{ What is the key factor affecting the effectiveness of PrEP? } \\
\hline Gender & 1 & 0.1 \\
\hline Age & 0 & 0 \\
\hline Adherence & 60 & 84.5 \\
\hline I do not know & 10 & 1.4 \\
\hline \multicolumn{3}{|c|}{$\begin{array}{l}\text { 'Alcohol should not be consumed while using PrEP'. Please } \\
\text { indicate whether this statement is correct or not. }\end{array}$} \\
\hline True & 42 & 59.2 \\
\hline False & 7 & 9.9 \\
\hline I do not know & 22 & 30.9 \\
\hline \multicolumn{3}{|c|}{$\begin{array}{l}\text { Has the Chinese Drug Administration approved tenofovir/ } \\
\text { emtricitabine as a PrEP drug? }\end{array}$} \\
\hline Yes & 23 & 32.4 \\
\hline No & 13 & 18.3 \\
\hline I do not know & 35 & 49.3 \\
\hline \multicolumn{3}{|c|}{ Have you ever taken PrEP pills? n=145 } \\
\hline No & 144 & 99.3 \\
\hline Yes & 1 & 0.7 \\
\hline \multicolumn{3}{|l|}{ View of PrEP $(n=255)$} \\
\hline Not sure & 11 & 4.3 \\
\hline $\begin{array}{l}\text { Not effective in HIV } \\
\text { prevention }\end{array}$ & 3 & 1.2 \\
\hline $\begin{array}{l}\text { May have some preventive } \\
\text { effect }\end{array}$ & 136 & 53.3 \\
\hline Very effective & 105 & 41.2 \\
\hline
\end{tabular}

$\mathrm{CBO}$, community-based organisation; PrEP, pre-exposure prophylaxis; VCT, voluntary counseling and testing.

PrEP (OR: $0.25,95 \%$ CI 0.12 to 0.54 ) would be less likely to use PrEP, compared with those who thought PrEP was very effective. But this association did not remain significant in the multivariable model when adjusted for other characteristics (table 5).

According to the multivariate logistic regression, having a higher education level (OR:0.29, 95\% CI 0.12 to 0.72 ), having told parents or siblings about one's sexual orientation (OR: 2.29, 95\% CI 0.97 to 5.39), being aware of PrEP (OR: $4.20,95 \%$ CI 1.64 to 10.73 ) and self-rating a high level of risk and fear of getting HIV (OR: 14.47, 95\% CI 2.19 to 95.53 ) were independently associated with willingness to use PrEP.

\section{DISCUSSION}

This is the first study to assess willingness to use PrEP and other associated factors among MSM in Hunan Province, China. The results of the present study suggest that the majority of MSM in Changsha City lack knowledge of PrEP. Participants had uncertainty about PrEP, including willingness to use PrEP and possible barriers they might face if they use PrEP. Only $15.3 \%$ of MSM were interested in using PrEP.

Willingness to use PrEP was relatively low among the MSM in this study. A global estimate of the acceptability of PrEP among MSM showed that acceptance ranged from $19.1 \%$ to $96.2 \%$ with a pooled estimate of $57.8 \%{ }^{27}$ The rate of willingness to use PrEP in the present study was lower than rates reported in Shanghai City $(19.1 \%)^{25}$; Guangzhou City $(36.2 \%)^{14}$; Chengdu City $(84.9 \%$ if PrEP was effective and free) ${ }^{15}$; Brazil, Mexico and Peru $(64.2 \%)^{28}$ and Kenya $(44.9 \%) .{ }^{29}$ There are several potential reasons for the low rate of willingness to use PrEP in the present sample. First, a lack of sufficient information and knowledge of PrEP could inhibit willingness to use PrEP. The present results showed that only $43.1 \%$ of the participants had ever heard of PrEP, and these participants had poor knowledge of PrEP. TDF/FTC was approved as PrEP for HIV prevention on 11 August 2020, ${ }^{11}$ while the present study was carried out in 2019. At the time of this study, there were no policies to support PrEP; even now, China has not yet issued any medical guidance for the use of PrEP. The inclusion of PrEP in standard strategies for HIV prevention still has a long way to go. Second, Changsha City is in the South-Central part of the Chinese mainland, and MSM in Changsha may receive less new and reliable information about PrEP and may be less open to new approaches to HIV prevention compared with MSM in some cities with more developed economies or more frequent international communications. Third, the measurement tool used in this study was a 19-item scale that asked participants to consider multiple circumstances related to taking PrEP. Unlike previous studies in China, which have typically asked participants to consider PrEP under ideal circumstances (eg, where PrEP is 100\% effective and free), the 19-item scale in this study asked participants to consider a broader set of issues. This may have resulted in lower level of but could be more trustworthy than those of previous studies by considering a range of issues that may affect willingness to use PrEP.

Cost may be a particular barrier to PrEP use. In this study, $33.7 \%$ of the participants were full-time students, without stable income and $48.2 \%$ indicated a monthly income of less than 4000 RMB (approximately US\$587). In Changsha, a bottle of TDF/FTC costs 1980 RMB (approximately US\$291). To access PrEP, they need to pay out-of-pocket costs for a medical examination before they receive a prescription and additional costs for routine check-ups. Thus, using PrEP would create a significant financial burden for low-income earners. Unfortunately, there is no sign that PrEP will be free or covered by health insurance in the next few years in China. 
Table 5 Logistic regression of willingness to use $\operatorname{PrEP}(n=255)$

\begin{tabular}{|c|c|c|c|c|c|}
\hline \multirow[b]{2}{*}{ Variable } & \multirow[b]{2}{*}{ Category } & \multicolumn{2}{|l|}{$\begin{array}{l}\text { Univariate logistic } \\
\text { regression }\end{array}$} & \multicolumn{2}{|c|}{ Multivariate logistic regression } \\
\hline & & OR $(95 \% \mathrm{Cl})$ & $\mathbf{P}$ & OR $(95 \% \mathrm{Cl})$ & $\mathbf{P}$ \\
\hline $\begin{array}{l}\text { Educational } \\
\text { background }\end{array}$ & University/college or above & $0.46(0.22$ to 0.94$)$ & 0.033 & $0.29(0.12$ to 0.72$)$ & 0.007 \\
\hline $\begin{array}{l}\text { Told parents or } \\
\text { siblings about sexual } \\
\text { orientation }\end{array}$ & No & Ref. & & & \\
\hline \multirow{4}{*}{$\begin{array}{l}\text { Self-perception of } \\
\text { risk of HIV infection }\end{array}$} & No risk & Ref. & & & \\
\hline & Low risk (<25.0\%) & NS & & & \\
\hline & Median risk (49.0 25.0\%) & NS & & & \\
\hline & High risk (25.0 50.0\%) & NS & & & \\
\hline \multirow{4}{*}{$\begin{array}{l}\text { Participants' view of } \\
\text { PrEP }\end{array}$} & Very effective & Ref. & & & \\
\hline & May have some preventive effect & NS & & & \\
\hline & May have no preventive effects & NS & & & \\
\hline & Not sure & $0.25(0.12$ to 0.54$)$ & $<0.001$ & NS & \\
\hline
\end{tabular}

NS, not significant in the analysis; PrEP, pre-exposure prophylaxis; Ref., reference.

According to our analysis, awareness of PrEP was 43.1\% and positively associated with willingness to use PrEP. This result was consistent with those of previous studies, which have indicated that increasing awareness of PrEP can be helpful in increasing the acceptance and use of PrEP. ${ }^{213031}$ One study revealed that higher levels of PrEP comprehension encouraged MSM in China to use PrEP under various conditions. ${ }^{14}$ Similarly, a Latin American study showed that PrEP use was directly related to PrEP awareness and that increasing awareness and knowledge of PrEP (including its safety and efficacy) were key to increasing the demand for PrEP. ${ }^{28}$ To promote PrEP programmes effectively, reliable information, targeted education and PrEP counselling should be delivered to key populations. A social network intervention in the USA indicated that after the provision of information to increase knowledge about PrEP's benefits, address concerns about PrEP and endorse PrEP use as a symbol of pride and health, the percentage of participants who reported using PrEP increased from $3 \%$ to $11 \% .{ }^{32}$ A study in North Brazil showed that participants who learnt about PrEP online were more willing to use PrEP. ${ }^{33}$ Educational strategies to raise awareness must be targeted to address specific barriers contributing to low levels of PrEP acceptability, such as doubts about its efficacy, side effects, perceived risk and access to PrEP services. ${ }^{33}$ To spread the use of PrEP in China, more correct information about PrEP should be provided to MSM.

Consistent with several previous studies, ${ }^{25}{ }^{3034-38}$ MSM in the present study who perceived themselves as being at a higher risk of HIV infection (vs no risk) were more willing to use PrEP. A study in California showed that those with medium and high levels of concern about contracting HIV were almost two times as likely to be willing to take PrEP. $^{39}$ As such, self-evaluation of HIV infection risk with professional guidance may potentially improve the uptake of PrEP and should be encouraged.

The present study showed that having told parents/ siblings about one's sexual orientation was associated with willingness to use PrEP. MSM who were comfortable about their sexual identities and willing to disclose them to their families were more likely to access social and emotional support. Previous studies have indicated that 'outness' is associated with higher access to HIV prevention services and sexual risk reduction. ${ }^{40-42}$

Some studies have found that PrEP willingness was positively associated with higher educational level. ${ }^{43-45}$ Conversely, a higher education level was associated with lower willingness to use PrEP in this study. A possible explanation could be that participants with more education were more concerned about PrEP's effectiveness, safety and side effects, so they were more cautious about PrEP use. ${ }^{46-49}$

Further interventions should be designed to provide information regarding PrEP to MSM. As internet access is now convenient, interventions to increase knowledge of PrEP could be achieved through web-based advertisements, online courses, digital counselling, peer education and community-based education. As primary hubs of disseminating healthcare information for MSM, CBOs should take responsibility for the delivery of information about PrEP and PrEP-related health services. 


\section{Limitations}

Several limitations of this study should be acknowledged. First, the participants in this study comprised a convenience sample of MSM connected to local HIV/AIDSfocused CBOs; thus, they were not representative of all MSM in China. As MSM visiting CBOs have more chance to learn about PrEP, their awareness of PrEP might have been higher than other MSM in China. Second, the selfreported nature of the questionnaire could have led to recall and social desirability biases, especially with regards to the data concerning risky sexual behaviours and drug use. To minimise recall bias, most questions were limited to behaviours and experiences occurring in the past 6 months. Third, this study did not meet the target sample size as one uncompleted questionnaire was removed. Finally, this cross-sectional study cannot be used to infer causality. Despite these limitations, the present study contributes to a growing body of literature on willingness to use PrEP and other associated factors, providing practical recommendations for the implementation of PrEP programmes in China.

\section{CONCLUSIONS}

The present study revealed relatively low awareness of and willingness to use PrEP among MSM in Changsha City, China. This study also investigated concerns about potential barriers to PrEP use. Awareness of PrEP, higher self-rated risk and fear of contracting HIV and sharing one's sexual orientation with parents or siblings were facilitators of willingness to use PrEP. Therefore, MSM should be educated on PrEP knowledge and how to selfevaluate their risk of HIV infection. Helping MSM to accept their sexual identities and providing them with more emotional and social support might improve PrEP acceptance. Interventions are required to increase awareness and knowledge of PrEP among both MSM and infectious disease specialists. Internet-based advertisements and programmes should be implemented to encourage MSM communities and MSM-friendly CBOs.

Acknowledgements First, I wish to thank all the survey participants. I also wish to thank the CBOs Zhong Da Yang Guang, Zuo An Cai Hong and Tian Kong Tong Lan for their assistance in the recruiting process and for providing their office rooms for researchers to communicate with the participants and collect information. I wish to thank Dr Martin Holt for permitting us to use his scale and answering our questions. Finally, I wish to thank my supervisor, Dr Honghong Wang, for proposing that I investigate PrEP and for her caring and encouragement.

Contributors SY collected the data, conducted the statistical analyses and wrote the paper. SY and HW contributed to the study design and to early drafts of the paper. Professor XL and KZ aided in the content validity index for scale development. WC, LLYL and BB reviewed and edited the manuscript. All authors agreed to the final content. HW is resonsible for the overall content as the guarantor. HW accepts full responsibility for the finished work and the conduct of the study, had access to the data, and controlled the decision to publish.

Funding This study was supported by the Philosophy and Social Science Foundation of Hunan Province (grant number 16YBA365). The funding body had no role in the design of the study; the collection, analysis and interpretation of the data; or the writing of the manuscript.

Competing interests None declared.
Patient and public involvement Patients and/or the public were not involved in the design, or conduct, or reporting, or dissemination plans of this research.

\section{Patient consent for publication Not applicable.}

Ethics approval Ethical approval was obtained from the Institutional Review Board of Xiangya Nursing School, Central South University, Changsha, China (number E201912). The essential information of the study, including its purpose and procedures, the benefits and risks for participants, and their right to decline participation in the study were clearly explained to the participants.

Provenance and peer review Not commissioned; externally peer reviewed.

Data availability statement Data are available upon reasonable request. The datasets used and/or analysed in the present study are available from the corresponding author upon reasonable request.

Open access This is an open access article distributed in accordance with the Creative Commons Attribution Non Commercial (CC BY-NC 4.0) license, which permits others to distribute, remix, adapt, build upon this work non-commercially, and license their derivative works on different terms, provided the original work is properly cited, appropriate credit is given, any changes made indicated, and the use is non-commercial. See: http://creativecommons.org/licenses/by-nc/4.0/.

\section{ORCID iDs}

Simin Yu http://orcid.org/0000-0003-2910-8712

Xianhong Li http://orcid.org/0000-0003-4063-4131

Honghong Wang http://orcid.org/0000-0002-3484-8929

\section{REFERENCES}

1 UNAIDS Data. Global HIV \& AIDS statistics - 2020 fact sheet, 2020. Available: https://www.unaids.org/en/resources/fact-sheet

2 Chinese Center for Disease Control and Prevention. New progress has been made in AIDS prevention and control in China in 2019, 2020. Available: http://www.nhc.gov.cn/jkj/s3586/201911/c2388ce7 Obdd404ea6dfcd886591784d.shtml

3 World Health Organization. Biobehavioural survey guidelines for populations at risk for HIV, 2020. Available: https://www.who.int/hiv/ pub/guidelines/biobehavioral-hiv-survey/en/

4 Yang XY. Practice and challenges of HIV pre-exposure prophylaxis and post-exposure prophylaxis. Chin J AIDS STD 2019;25:425-8.

5 World Health Organization. Guidance on oral pre-exposure prophylaxis (PreP) for serodiscordant couples, men and transgender women who have sex with men at high risk of HIV: recommendations for use in the context of demonstration projects, 2020. Available: https://www.who.int/hiv/pub/guidance_prep/en/

6 World Health Organization. Guideline on when to start antiretroviral therapy and on pre-exposure prophylaxis for HIV, 2020. Available: https://www.who.int/hiv/pub/guidelines/earlyrelease-arv/en/

7 World Health Organization. What's the 2+1+1? Event-driven oral preexposure prophylaxis to prevent HIV for men who have sex with men: Update to WHO's recommendation on oral PrEP, 2020. Available: https://www.who.int/hiv/pub/prep/211/en/

8 PrEP Watch. Country updates 2020, 2020. Available: https://www. prepwatch.org/in-practice/country-updates/

9 Joint United Nations Program on HIV/AIDS (UNAIDS). Miles to goclosing gaps, breaking barriers, righting injustices, 2020. Available: https://www.unaids.org/en/resources/documents/2018/global-aidsupdate

10 Chinese Center for Disease Control and Prevention. Implementation of the program to stop the spread of AIDS (2019-2022), 2020. Available: http://www.nhc.gov.cn/jkj/s7925/201910/adc374d06131 44b2b7bb5d6c58a60223.shtml

11 Gilead Sciences. Chinese national medical products administration approves Truvada $₫$ for HIV pre-exposure prophylaxis (PreP), 2020. Available: https://www.gileadchina.com/news/press-releases/2020/8/ china-national-medical-products-administration-approves-truvada

12 Zhou F, Gao L, Li S, et al. Willingness to accept HIV pre-exposure prophylaxis among Chinese men who have sex with men. PLoS One 2012;7:e32329.

13 Zhang Y, Peng B, She Y, et al. Attitudes toward HIV pre-exposure prophylaxis among men who have sex with men in Western China. AIDS Patient Care STDS 2013;27:137-41.

14 Zheng Z-W, Qiu J-L, Gu J, et al. Preexposure prophylaxis comprehension and the certainty of willingness to use preexposure prophylaxis among men who have sex with men in China. Int J STD AIDS 2019;30:4-11.

15 Peng L, Cao W, Gu J, et al. Willingness to use and adhere to HIV pre-exposure prophylaxis (PreP) among men who have sex with men (MSM) in China. Int J Environ Res Public Health 2019;16:2620. 
16 Han J, Bouey JZ, Wang L, et al. Prep uptake preferences among men who have sex with men in China: results from a national Internet survey. J Int AIDS Soc 2019;22:e25242.

17 Wang Z, Lau JTF, Fang Y, et al. Prevalence of actual uptake and willingness to use pre-exposure prophylaxis to prevent HIV acquisition among men who have sex with men in Hong Kong, China. PLoS One 2018;13:e0191671.

18 Wang X, Bourne A, Liu P, et al. Understanding willingness to use ora pre-exposure prophylaxis for HIV prevention among men who have sex with men in China. PLoS One 2018;13:e0199525.

19 Xie PY, Wang X, Liu PL, et al. [Willingness and influencing factors of using pre-exposure prophylaxis among 301 men have sex with men in Wuhan city, 2015]. Zhonghua Yu Fang Yi Xue Za Zhi 2017;51:1001-6.

20 Mao X, Yu H, Hu QH, et al. [Acceptability of pre-exposure HIV prophylaxis clinical trial among MSM in Shenyang city]. Zhonghua Liu Xing Bing Xue Za Zhi 2017;38:1083-7.

21 Lee $\mathrm{Y}-\mathrm{C}$, Chang S-Y, Lin K-Y, et al. Awareness and willingness towards pre-exposure prophylaxis against HIV infection among individuals seeking voluntary counselling and testing for HIV in Taiwan: a cross-sectional questionnaire survey. BMJ Open 2017;7:e015142.

22 Ko N-Y, Chen B-J, Li C-W, NY K, CW L, et al. Willingness to self-pay for pre-exposure prophylaxis in men who have sex with men: a national online survey in Taiwan. AIDS Educ Prev 2016;28:128-37.

23 Cao W, Sun S, Peng L, et al. Low willingness to pay for pre-exposure prophylaxis (PreP) among men who have sex with men (MSM) in China. BMC Public Health 2020;20:258-63.

24 Ding Y, Yan H, Ning Z, et al. Low willingness and actual uptake of pre-exposure prophylaxis for HIV-1 prevention among men who have sex with men in Shanghai, China. Biosci Trends 2016;10:113-9.

25 Holt M, Murphy DA, Callander D. Willingness to use HIV preexposure prophylaxis and the likelihood of decreased condom use are both associated with unprotected anal intercourse and the perceived likelihood of becoming HIV positive among Australian gay and bisexual men. Sex Transm Infect.

26 Shi J, Mo X, Sun Z. [Content validity index in scale development]. Zhong Nan Da Xue Xue Bao Yi Xue Ban 2012;37:49-52.

27 Peng P, Su S, Fairley CK, et al. A global estimate of the acceptability of pre-exposure prophylaxis for HIV among men who have sex with men: a systematic review and meta-analysis. AIDS Behav 2018;22:1063-74.

28 Torres TS, Konda KA, Vega-Ramirez EH, et al. Factors associated with willingness to use pre-exposure prophylaxis in Brazil, Mexico, and Peru: web-based survey among men who have sex with men. JMIR Public Health Surveill 2019;5:e13771.

29 Ogunbajo A, Kang A, Shangani S, et al. Awareness and acceptability of pre-exposure prophylaxis (PreP) among gay, bisexual and other men who have sex with men (GBMSM) in Kenya. AIDS Care 2019;31:1185-92.

30 Goedel WC, Halkitis PN, Greene RE, et al. Correlates of awareness of and willingness to use pre-exposure prophylaxis (PreP) in gay, bisexual, and other men who have sex with men who use geosocialnetworking smartphone applications in New York City. AIDS Behav 2016;20:1435-42.

31 Ferrer L, Folch C, Fernandez-Davila P, et al. Awareness of preexposure prophylaxis for HIV, willingness to use it and potential barriers or facilitators to uptake among men who have sex with men in Spain. AIDS Behav 2016;20:1423-33.

32 Kelly JA, Amirkhanian YA, Walsh JL, et al. Social network intervention to increase pre-exposure prophylaxis (PreP) awareness, interest, and use among African American men who have sex with men. AIDS Care 2020;32:40-6.
33 Magno L, Dourado I, Sutten Coats C, et al. Knowledge and willingness to use pre-exposure prophylaxis among men who have sex with men in northeastern Brazil. Glob Public Health 2019:14:1098-111.

34 Holt M, Lea T, Murphy D, et al. Willingness to use HIV pre-exposure prophylaxis has declined among Australian gay and bisexual men: results from repeated national surveys, 2011-2013. J Acquir Immune Defic Syndr 2014;67:222-6.

35 Krakower DS, Mimiaga MJ, Rosenberger JG, et al. Limited awareness and low immediate uptake of pre-exposure prophylaxis among men who have sex with men using an Internet social networking site. PLoS One 2012;7:e33119.

36 Wilton J, Kain T, Fowler S, et al. Use of an HIV-risk screening tool to identify optimal candidates for PreP scale-up among men who have sex with men in Toronto, Canada: disconnect between objective and subjective HIV risk. J Int AIDS Soc 2016;19:20777.

37 McMahan VM, Moreno C, Al-Tayyib A, et al. Pre-Exposure prophylaxis awareness and use among cisgender men who have sex with men and use methamphetamine in 3 Western us cities. Sex Transm Dis 2020;47:217-23.

38 Torres TS, Luz PM, De Boni RB, et al. Factors associated with PrEP awareness according to age and willingness to use HIV prevention technologies: the 2017 online survey among MSM in Brazil. AIDS Care 2019;31:1193-202.

39 Holloway IW, Tan D, Gildner JL, et al. Facilitators and barriers to preexposure prophylaxis willingness among young men who have sex with men who use geosocial networking applications in California. AIDS Patient Care STDS 2017;31:517-27.

40 White D, Stephenson R. Identity formation, outness, and sexual risk among gay and bisexual men. Am J Mens Health 2014;8:98-109.

41 Pitpitan EV, Smith LR, Goodman-Meza D, et al. "Outness" as a moderator of the association between syndemic conditions and HIV risk-taking behavior among men who have sex with men in Tijuana, Mexico. AIDS Behav 2016;20:431-8.

42 Woodward EN, Banks RJ, Marks AK, et al. Identifying resilience resources for HIV prevention among sexual minority men: a systematic review. AIDS Behav 2017;21:2860-73.

43 Holt M, Lea T, Schmidt H-M, et al. Willingness to use and have sex with men taking HIV pre-exposure prophylaxis (PrEP): results of online surveys of Australian gay and bisexual men, 2011-2015. Sex Transm Infect 2017;93:438-44.

44 Hoots BE, Finlayson T, Nerlander L, et al. Willingness to take, use of, and indications for pre-exposure prophylaxis among men who have sex with Men-20 us cities, 2014. Clin Infect Dis 2016;63:672-7.

$45 \mathrm{Fu}$ Y, Ashuro AA, Feng X, et al. Willingness to use HIV pre-exposure prophylaxis and associated factors among men who have sex with men in Liuzhou, China. AIDS Res Ther 2021;18:46.

46 Liu AY, Kittredge PV, Vittinghoff E, et al. Limited knowledge and use of HIV post- and pre-exposure prophylaxis among gay and bisexual men. J Acquir Immune Defic Syndr 2008;47:241-7.

47 Mimiaga MJ, Case P, Johnson CV, et al. Preexposure antiretroviral prophylaxis attitudes in high-risk Boston area men who report having sex with men: limited knowledge and experience but potential for increased utilization after education. $J$ Acquir Immune Defic Syndr 2009;50:77-83.

48 Yan Z, Zhong X, Peng B, et al. Awareness, experience and willingness of using HIV pre-exposure prophylaxis among men who have sex with men. J Third Mil Med Univ 2013;35:2226-30.

49 Can X, Chaozhi F, Jing M. Willingness to use pre-exposure prophylaxis among 200 men who have sex with men in Kaiyuan City. Chinese Journal of AIDS \& STD 2019;25:741-2. 